\title{
Estimating the Size of the Image of Deterministic Hash Functions to Elliptic Curves
}

\author{
Pierre-Alain Fouque and Mehdi Tibouchi ${ }^{\star}$ \\ École normale supérieure \\ Département d'informatique, équipe de cryptographie \\ 45, rue d'Ulm, F-75230 Paris Cedex 05, France \\ \{pierre-alain.fouque, mehdi.tibouchi\}@ens.fr
}

\begin{abstract}
Let $E$ be a non-supersingular elliptic curve over a finite field $\mathbb{F}_{q}$. At CRYPTO 2009, Icart introduced a deterministic function $\mathbb{F}_{q} \rightarrow$ $E\left(\mathbb{F}_{q}\right)$ which can be computed efficiently, and allowed him and Coron to define well-behaved hash functions with values in $E\left(\mathbb{F}_{q}\right)$. Some properties of this function rely on a conjecture which was left as an open problem in Icart's paper. We prove this conjecture below as well as analogues for other hash functions.
\end{abstract}

Keywords: Elliptic Curves, Function Fields, Hash Functions.

\section{Introduction}

In cryptography, it has been an open problem for a long time to transform a random value in $\mathbb{F}_{q}$ into a random point on an elliptic curve in a deterministic and efficient manner. Such transformations $f$ are called hash functions since they have been used, say in the context of identity-based encryption $[1,8]$, by first hashing an identity into a random value in $\mathbb{F}_{q}$ using a standard cryptographic hash function $h$ and then applying such a transformation to get a point on the curve: $H(m)=f(h(m))$. They have also applications in password-based authentication schemes [3]. However, only probabilistic solutions were known before 2006 .

The usual solution before 2006 was to take $x \in \mathbb{F}_{q}$ and check whether this value corresponds to a valid abscissa of a point on the elliptic curve. If not, try another abscissa until one of them works. Consequently, random bits are needed to perform this random search and the running time cannot be bounded and cannot be constant. The main drawback of this approach is that for password-based authentication schemes, an adversary can perform timing attacks and off-line computations in exhaustive

\footnotetext{
* This research was carried out while the second author was visiting the Okamoto Research Laboratory at the NTT Information Sharing Platform (Tokyo, Japan).
} 
search attacks [2]. Some passwords do not need to be tested if the number of iterations of the probabilistic process is not the correct one. Indeed, security proofs for password-based authentication schemes rely on the fact that only on-line attacks are possible and each try allows to remove a small constant number of passwords, ideally one. Other cryptographic solutions have been proposed to avoid the random process but they made the protocol more complex. One of these is to apply the protocol twice, once with the original curve and in parallel on one of the twisted curves of the original curve. Now, any value in $\mathbb{F}_{q}$ corresponds either to an abscissa of the original curve or of the associated twisted curve since the two curves represent a distinct union of $\mathbb{F}_{q}$. Finally, it is worth noticing that the function $h(m) \cdot G$ were $G$ is a generator of the point group of the curve is not a secure solution since the discrete log of the point is known and this makes most protocols insecure.

Deterministic functions. To construct such function, Shallue and van de Woestijne at ANTS 2006 [12] proposed a deterministic algorithm based on Skalba's inequality. The running time of this function is $O\left(\log ^{4} q\right)$. Later, a generalization for hyper-elliptic curve was proposed by Ulas [14]. At CRYPTO 2009, Icart [10] proposed another more efficient technique in $O\left(\log ^{3} q\right)$. Finally, Brier et al. [4] propose another technique based on a variant of the Shallue-Woestijne-Ulas (SWU) function, and explain how to construct secure hash functions to elliptic curves based on Icart's function or SWU.

Ideally, it would be nice if the image of the hash function was the whole curve, and if the distribution on the points was statistically close to uniform. In order to prove such results, it is interesting to know how many points there are in the image. Icart showed a coarse bound for his function $f: q / 4 \leq \# f\left(\mathbb{F}_{q}\right) \leq q$. He conjectured that

$$
\left|\# f\left(\mathbb{F}_{q}\right)-\frac{5 q}{8}\right| \leq \lambda \sqrt{q}
$$

for some constant $\lambda$ but left this conjecture as an open problem. Similar statements can be formulated about the size of the image of other hash functions, such as the characteristic 2 version of Icart's function, or the simplified version of SWU proposed by Brier et al..

Related Work. Very recently and independently of our work, Farashahi, Shparlinski and Voloch have also analyzed Icart's function in the case of 
finite fields of odd characteristic, using Chebotarev theorem in [9] as we did. However, our paper also covers the case of even characteristic for Icart function which is interesting in a cryptographic point of view and the simplified version of SWU which is important in the new paper of Brier et al. [4].

It is interesting to note that, depending on the particular function we consider, the number of points in the image varies according to some Galois group associated with the function.

Organization of the paper. In section 2, we describe Icart's hash function and his conjecture. Then, we prove the conjecture for curves of odd characteristic, of characteristic 2 and finally for the variant of SWU.

\section{Preliminaries}

\subsection{Icart's function}

Let $\mathbb{F}_{q}$ be a finite field of characteristic $>3$ and $E$ an elliptic curve over $\mathbb{F}_{q}$. $E$ can be represented as the union of its neutral element $O$ and the set of points $(x, y)$ in the affine plane over $\mathbb{F}_{q}$ such that:

$$
y^{2}=x^{3}+a x+b
$$

for some suitable constants $a, b \in \mathbb{F}_{q}$ satisfying $4 a^{3}+27 b^{2} \neq 0$ (nonsingularity).

When $q-1$ is not divisible by 3 , these curves are supersingular for $a=0$. In all other cases, Icart [10] defines the following function $f_{a, b}: \mathbb{F}_{q} \rightarrow$ $E\left(\mathbb{F}_{q}\right)$. He sets $f_{a, b}(0)=O$ and for all $u \neq 0, f_{a, b}(u)=(x, y)$ with:

$$
\begin{aligned}
& x=\left(v^{2}-b-\frac{u^{6}}{27}\right)^{1 / 3}+\frac{u^{2}}{3} \\
& y=u x+v
\end{aligned}
$$

where $v=\left(3 a-u^{4}\right) /(6 u)$. This function is shown to be well-defined and easily computed in deterministic polynomial time. Moreover, if $(x, y)$ is a point in $E\left(\mathbb{F}_{q}\right)$, then $f_{a, b}(u)=(x, y)$ if and only if $u$ satisfies the quartic equation

$$
u^{4}-6 x u^{2}+6 y u-3 a=0
$$




\subsection{Icart's conjecture}

In [10], Icart conjectures that the image of $f_{a, b}$ contains $(5 / 8) \cdot \# E\left(\mathbb{F}_{q}\right)+$ $O\left(q^{1 / 2}\right)$ points of the curve. In view of the previous equation, and since the curve itself has $\# E\left(\mathbb{F}_{q}\right)=q+O\left(q^{1 / 2}\right)$ points in $\mathbb{F}_{q}$, this conjecture can be stated as follows.

Conjecture 1 (Icart). Let $K=\mathbb{F}_{q}(x, y)=\mathbb{F}_{q}(x)[Y] /\left(Y^{2}-x^{3}-a x-b\right)$ be the function field of $E$, and $P$ the polynomial in $K[u]$ defined by $P(u)=u^{4}-6 x u^{2}+6 y u-3 a$. Let further $N$ be the number of points in $E\left(\mathbb{F}_{q}\right)$ at which the reduction of $P$ has a root in $\mathbb{F}_{q}$. Then

$$
N=\frac{5}{8} q+O\left(q^{1 / 2}\right)
$$

The next section is devoted to the proof of this conjecture.

\section{Proof of Icart's conjecture}

\subsection{Genericity of $P$}

Proposition 1. Let again $K=\mathbb{F}_{q}(x, y)$ be the function field of $E$. The polynomial $P(u)=u^{4}-6 x u^{2}+6 y u-3 a \in K[u]$ is irreducible over $K$, and its Galois group is $S_{4}$.

Proof. Introduce the resolvent cubic of $P$, whose roots in an algebraic closure are $\left(r_{i}+r_{j}\right)\left(r_{k}+r_{l}\right)$ for all permutations $(i, j, k, l)$ of $(1,2,3,4)$, with $r_{1}, \ldots, r_{4}$ the roots of $P:^{1}$

$$
\begin{aligned}
C(u) & =u^{3}+12 x u^{2}+\left(36 x^{2}+12 a\right) u+36 y^{2} \\
& =u^{3}+12 x u^{2}+\left(36 x^{2}+12 a\right) u+36\left(x^{3}+a x+b\right) \in \mathbb{F}_{q}(x)
\end{aligned}
$$

According to classical facts about the quartic equation (see Appendix A), it suffices to prove that $P$ and $C$ are irreducible over $K$, and that their common discriminant

$$
\Delta=-432\left(9 x^{6}+18 a x^{4}+90 b x^{3}-39 a^{2} x^{2}-54 a b x+16 a^{3}+81 b^{2}\right)
$$

is not a square in $K$. Moreover, we can prove these assertions after extending the field of scalars to $F=\overline{\mathbb{F}}_{q}$. Indeed, if they hold over $F$, they clearly hold a fortiori over $\mathbb{F}_{q}$. The following three lemmas conclude the proof.

\footnotetext{
${ }^{1}$ Some texts use the resolvent whose roots are the $r_{i} r_{j}+r_{k} r_{l}$. This is of course equivalent, as both sets of roots have the same Galois action.
} 
Lemma 1. The resolvent cubic $C(u)$ is irreducible over $F(x, y)$.

Proof. This amounts to showing that $C(u)$ has no root in $F(x, y)$. Note first that it is actually sufficient to prove it has no root in $F(x)$. Indeed, if it is irreducible in $F(x)$ but has a root in $F(x, y)$, the degree of the algebraic extension $F(x, y) / F(x)$ must be divisible by $\operatorname{deg} C(u)=3$. But this extension is quadratic: hence a condraction.

Let then $f / g$ be a root of $C$ in $F(x)$, with $f$ and $g$ coprime polynomials. Multiplying the equation $C(f / g)=0$ by $g^{3}$, we get

$$
f^{3}=g \cdot\left(-12 x f^{2}-\left(36 x^{2}+12 a\right) f g-36\left(x^{3}+a x+b\right) g^{2}\right)
$$

Thus $g$ divides $f^{3}$, and since it is coprime to $f$, it must be constant. Without loss of generality, we thus have $g=1$ and

$$
f^{3}+12 x f^{2}+\left(36 x^{2}+12 a\right) f+36\left(x^{3}+a x+b\right)=0
$$

Let $m=\operatorname{deg} f$. Then the terms in the previous sum are of respective degrees $3 m, 2 m+1, m+2,3$. If $m \geq 2$, the sum is thus of degree $3 m$, and if $m \leq 0$, it is of degree 3 : in neither case can it be 0 . The only possibility is thus $m=1$ and $f=\alpha x+\beta$. We get

$$
\begin{gathered}
\left(\alpha^{3}+12 \alpha^{2}+36 \alpha+36\right) x^{3}+3 \beta\left(\alpha^{2}+8 \alpha+12\right) x^{2}+ \\
\left(3 \alpha \beta^{2}+12 a \alpha+12 \beta^{2}+36 a\right) x+\left(\beta^{3}+12 a \beta+36 b\right)=0
\end{gathered}
$$

in $F(x)$. Suppose $\beta \neq 0$. Since the coefficients of $x^{3}$ and $x^{2}$ must be zero, this gives $\alpha^{3}+12 \alpha^{2}+36 \alpha+36=\alpha^{2}+8 \alpha+12=0$, which is impossible, since the polynomials $X^{3}+12 X^{2}+36 X+36$ and $X^{2}+8 X+12$ are coprime. Hence $\beta=0$, and thus $\alpha^{3}+12 \alpha^{2}+36 \alpha+36=12 a(\alpha+3)=0$, which is similarly seen to be impossible (as $a \neq 0$ ). This completes the proof.

Lemma 2. The discriminant $\Delta$ is not a square in $F(x, y)$.

Proof. Again, we will show that it is sufficient to prove that $\Delta$ is not a square in $F(x)$. Indeed, suppose that $\Delta$ is not a square in $F(x)$ but becomes a square in $F(x, y)$. Since the extension is quadratic, this gives $F(x, y)=F(x, \sqrt{\Delta})$. In particular, if $\lambda$ is a root of $X^{3}+a X+b$ in $F$, the extension $F(x, \sqrt{\Delta}) / F(x)$ must be ramified at $(x-\lambda)$. In other words, if we specialize $\Delta(x)$ at $x=\lambda$, we must get 0 . But

$$
\begin{aligned}
(\lambda-3 b / a) \Delta(\lambda) & =16 \cdot 432(\lambda-3 b / a)\left(3 a^{2} \lambda^{2}+9 a b \lambda-a^{3}\right) \\
& =16 \cdot 432\left[3 a^{2}\left(\lambda^{3}+a \lambda+b\right)-\left(4 a^{3}+27 b^{2}\right) \lambda\right] \\
& =-16 \cdot 432\left(4 a^{3}+27 b^{2}\right) \lambda \neq 0
\end{aligned}
$$


since the characteristic does not divide 6 and $a \neq 0$. Hence a contradiction.

It remains to prove that $\Delta$ is not a square in $F(x)$, or equivalently in $F[x]$ (since $F[x]$ is integrally closed). A square root of $\Delta$ in $F[x]$ must have the form $S=\sqrt{-432} \cdot\left(3 x^{3}+r x^{2}+s x+t\right)$. The coefficient of $x^{5}$ in $S^{2}$ must be 0 , hence $r=0$. The coefficient of $x^{4}$ must be $18 a$, hence $s=3 a$. But then the coefficient of $x^{2}$ is equal to both $9 a^{2}$ and $-39 a^{2}$, which is a contradiction since $48 a^{2} \neq 0$. This completes the proof.

Lemma 3. The polynomial $P$ is irreducible over $F(x, y)$.

Proof. Let $\sigma$ be the non trivial Galois automorphism of the extension $F(x, y) / F(x)(\sigma(y)=-y)$. If $P(u)$ decomposes as a product of non constant factors in $F(x, y)[u]$, then its norm $P_{0}(u)=P(u) P(u)^{\sigma}$ is reducible over $F(x)$. We will show that this is not the case. Note first that $P_{0}(u)$ can be written as $Q_{0}\left(u^{2}\right)$, where

$$
Q_{0}(v)=v^{4}-12 x v^{3}+\left(36 x^{2}-6 a\right) v^{2}-36\left(x^{3}+b\right) v+9 a^{2}
$$

Now $Q_{0}(v)$ is easily seen to be an irreducible polynomial of $F(x)[v]$. Indeed, if it had a root $f / g \in F(x)$, the rational function $f / g$ would be constant, which is clearly impossible. And if it decomposes as a product of degree 2 factors $Q_{0}=\left(v^{2}+r v+s\right)\left(v^{2}+r^{\prime} v+s^{\prime}\right)$, these factors are in $F[x]$ (integrally closed domain). Since $s s^{\prime}=9 a^{2}$, both $s$ and $s^{\prime}$ are constant. Then, since the coefficient of $v^{2}, r r^{\prime}+s+s^{\prime}$, is of degree $2, r$ and $r^{\prime}$ are both of degree at most 2. But then so is $r s^{\prime}+r^{\prime} s$, which is the coefficient of $v$ in $Q_{0}$, namely $-36\left(x^{3}+b\right)$, hence a contradiction.

Now let $w$ be a root of $P_{0}$ in the separable closure of $F(x)$, and let $L=F(x, w), L^{\prime}=F\left(x, w^{2}\right) . L^{\prime}$ is a subfield of $L$, and a rupture field of $Q_{0}$. In particular $[L: F(x)]=\left[L: L^{\prime}\right] \cdot\left[L^{\prime}: F(x)\right]=4\left[L: L^{\prime}\right]$. Since the polynomial $P_{0}$ is even, $-w$ is another root of $P_{0}$. As $w \notin F(x), w \mapsto-w$ defines a non trivial $F(x)$-automorphism of $L$. This automorphism fixes $L^{\prime}$, so $\left[L: L^{\prime}\right] \geq 2$. This gives $[L: F(x)] \geq 8$, and thus $P_{0}$ must have an irreducible factor of degree $\geq 8$. In other words, $P_{0}$ is irreducible over $F(x)$ as required.

\subsection{Applying Chebotarev}

Now that Proposition 1 is established, Conjecture 1 readily follows from effective versions of the Chebotarev Density Theorem for function fields. One such version is [7, Proposition 6.4.8], from which one can easily deduce: 
Theorem 1 (Chebotarev). Let $K$ be an extension of $\mathbb{F}_{q}(x)$ of degree $d<\infty$ and $L$ a Galois extension of $K$ of degree $m<\infty$. Assume $\mathbb{F}_{q}$ is algebraically closed in $L$, and fix some subset $\mathscr{S}$ of $\operatorname{Gal}(L / K)$ stable under conjugation. Let $s=\# \mathscr{S}$ and $N(\mathscr{S})$ the number of places $v$ of $K$ of degree 1 , unramified in $L$, such that the Artin symbol $\left(\frac{L / K}{v}\right)$ (defined up to conjugation) is in $\mathscr{S}$. Then

$$
\left|N(\mathscr{S})-\frac{s}{m} q\right| \leq \frac{2 s}{m}\left(\left(m+g_{L}\right) \cdot q^{1 / 2}+m\left(2 g_{K}+1\right) \cdot q^{1 / 4}+g_{L}+d m\right)
$$

where $g_{K}$ and $g_{L}$ are the genera of the function fields $K$ and $L$.

Proof (of Conjecture 1). In our case, $K$ is the function field of $E$ and $L$ the splitting field of $P(u)$. In particular, $d=2, m=\# S_{4}=24$ and $g_{K}=1$. We consider the subset $\mathscr{S} \subset \operatorname{Gal}(L / K)=S_{4}$ consisting of permutations with at least one fixed point - these are the conjugates of (1), (12) and (123), and there are $s=1+6+8=15$ of them. Hence $s / m=15 / 24=5 / 8$.

The places $v$ of $K$ of degree 1 correspond to points of $E\left(\mathbb{F}_{q}\right)$ (in the projective plane), and for a point $\left(x_{0}, y_{0}\right) \in E\left(\mathbb{F}_{q}\right)$ not at infinity, saying that $v=\left(x-x_{0}\right)$ has its Artin symbol in $\mathscr{S}$ means that the reduction of $P(u)$ at $\left(x_{0}, y_{0}\right)$ is a polynomial over $\mathbb{F}_{q}$ which decomposes into a products of factors at least one of which is of degree 1 (it splits completely if the symbol is (1), decomposes as two linear factors and a quadratic if it is (12) and a product of a linear factor and a cubic if it is (123) up to conjugation).

In other words, $N(\mathscr{S})$ is the same as $N$ in the statement of Conjecture 1 up to a constant number accounting for ramified places (at most 12 since $\Delta$ is a polynomial of degree 6 in $x$ ) and the point at infinity. We then get

$$
\left|N-\frac{5}{8} q\right| \leq \frac{5}{4}\left(\left(24+g_{L}\right) \cdot q^{1 / 2}+72 q^{1 / 4}+g_{L}+48\right)+12+1
$$

To bound $g_{L}$, note again that there are at most 12 ramified points, and the ramification index is at most $\operatorname{deg} P_{0}=4$ at each of them. The RiemannHurwitz formula thus gives

$$
2-2 g_{L} \geq 24\left(2-2 g_{K}\right)-12 \cdot(4-1) \quad \text { i.e. } \quad g_{L} \leq 17
$$

and thus

$$
\left|N-\frac{5}{8} q\right| \leq \frac{5}{4}\left(41 q^{1 / 2}+72 q^{1 / 4}+76\right)
$$


In particular, $N=(5 / 8) q+O\left(q^{1 / 2}\right)$. Concretely, for all $q \geq 2^{19}$, we have

$$
\left|N-\frac{5}{8} q\right| \leq 55 q^{1 / 2}
$$

\section{Analogue in Characteristic 2}

In [10], Icart also introduces a variant of his function for elliptic curves over finite fields $\mathbb{F}_{q}$ of even characteristic, i.e. $q=2^{n}$. Such an elliptic curve has the form

$$
y^{2}+x y=x^{3}+a x^{2}+b
$$

with $a, b \in \mathbb{F}_{q}, b \neq 0$. Icart's function for such a curve $E$ is defined when $n$ is odd as

$$
\begin{aligned}
f_{a, b}: \mathbb{F}_{q} & \rightarrow E\left(\mathbb{F}_{q}\right) \\
u & \mapsto\left(x, u x+v^{2}\right)
\end{aligned}
$$

where $v=a+u+u^{2}$ and $x=\left(v^{4}+v^{3}+b\right)^{1 / 3}+v$. It is shown that $u \in \mathbb{F}_{q}$ maps to $(x, y) \in E\left(\mathbb{F}_{q}\right)$ if and only if $P(u)=0$, where $P \in K[u]$ is defined as

$$
P(u)=u^{4}+u^{2}+x u+\left(a^{2}+y\right)
$$

Using this result, we can prove the following analogue of Icart's conjecture.

Proposition 2. The number of points $N$ in the image of $f_{a, b}$ satisfies:

$$
N=\frac{5}{8} q+O\left(q^{1 / 2}\right)
$$

where the implied constant in the big-O is universal.

The proof is identical to the one in $\S 3.2$. The only difference is that the computation of the Galois group is slightly different in even characteristic. However, the group is still $S_{4}$. Let us prove this fact now.

Proposition 3. The polynomial $P(u)=u^{4}+u^{2}+x u+\left(a^{2}+y\right) \in K[u]$ is separable and irreducible over $K$, and its Galois group is $S_{4}$.

Proof. Since $P^{\prime}=x$ is a unit in $K[u], P$ is certainly separable. Thus, if we prove that it is irreducible, its Galois group can be determined according to [5, Theorem 3.4]: to see that it is $S_{4}$, it suffices to show that both its resolvent cubic $C$ and its resolvent quadratic $Q$ are irreducible (see Appendix A). 
First, we have $C(u)=u^{3}+u^{2}+x^{2}$. If this polynomial had a root in $\mathbb{F}_{q}(x)$, it would be a polynomial of $\mathbb{F}_{q}[x]$ dividing $x^{2}$ by integral closure, which is clearly impossible. Therefore, $C(u)$ is irreducible over $\mathbb{F}_{q}(x)$, and also over $K$ by the same degree argument as in the proof of Lemma 1: namely, if $C(u)$ had a root in $K,\left[K: \mathbb{F}_{q}(x)\right]=2$ would be divisible by $\operatorname{deg} C(u)=3$, a contradiction.

Additionally, we have $Q(u)=u^{2}+x^{2} u+x^{2}+x^{4}$. If $Q$ is reducible over $\mathbb{F}_{q}(x)$, we see again that it is split over $\mathbb{F}_{q}[x]$, and its roots $s, t$ satisfy $\operatorname{deg} s+\operatorname{deg} t=4$ and $\operatorname{deg}(s t)=2$, hence $s$ and $t$ are quadratics dividing $x^{2}(1+x)^{2}$, i.e. constant multiples of $x^{2}, x^{2}+x$ or $x^{2}+1$, but no such quadratic is a root of $Q$. Thus, $Q$ is irreducible over $\mathbb{F}_{q}(x)$. To see that it remains irreducible over $K$, it suffices to see that $K$ and the splitting field $F$ of $Q$ over $\mathbb{F}_{q}(x)$ are linearly disjoint, and this is certainly the case since $F$ is a function field of genus 0 whereas $K$ is of genus 1 .

Finally, let us prove that $P$ is irreducible. Let first $\sigma$ be the nontrivial Galois automorphism of $K / \mathbb{F}_{q}(x)$, namely $y \mapsto y+x$, and set $P_{0}=P P^{\sigma} \in \mathbb{F}_{q}(x)$. It suffices to prove that $P_{0}$ is irreducible over $\mathbb{F}_{q}(x)$. We have

$P_{0}=\left(u^{8}+u^{4}\right)+x\left(u^{4}+u^{2}\right)+x^{2}\left(u^{2}+u\right)+\left(x^{3}+a^{2} x^{2}+a^{2} x+a^{4}+b\right)=Q_{0}\left(u^{2}+u\right)$ where $Q_{0}(v)=v^{4}+x v^{2}+x^{2} v+\left(x^{3}+a^{2} x^{2}+a^{2} x+a^{4}+b\right)$.

If $Q_{0}$ has a root over $\mathbb{F}_{q}(x)$, it is in fact in $\mathbb{F}_{q}[x]$, which is not possible by inspection of the degrees of the four terms in the sum. Similarly, if $Q_{0}$ can be written as a product of factors of degree 2 , we have $Q_{0}=$ $\left(v^{2}+r+s\right)\left(v^{2}+r+s^{\prime}\right)$ with $r, s$ and $s^{\prime}$ are all in $\mathbb{F}_{q}[x]$ (with $r$ appearing in both factors by inspection of the degree 3 coefficient of $Q_{0}$ ). We get $\operatorname{deg}\left(s s^{\prime}\right)=3$, so the polynomial $s+s^{\prime}$ must be of degree at least 2 . Since $r\left(s+s^{\prime}\right)=x^{2}$, this implies that $r$ is constant. But then the relation $s+s^{\prime}+r^{2}=x$ gives a contradiction. Therefore $Q_{0}$ is irreducible over $\mathbb{F}_{q}(x)$.

Then, let $w$ be a root of $P_{0}$ in the separable closure of $\mathbb{F}_{q}(x)$, and set $L=\mathbb{F}_{q}(x, w), L^{\prime}=\mathbb{F}_{q}\left(x, w+w^{2}\right)$. Like in the proof of Lemma 3, we have a tower of extensions $\mathbb{F}_{q}(x) \subset L^{\prime} \subset L$, and $L^{\prime}$ is a rupture field of $Q_{0}$, so $\left[L: \mathbb{F}_{q}(x)\right]=4\left[L: L^{\prime}\right]$. Furthermore, since $P_{0}(u+1)=P(u), w \mapsto w+1$ is a non-trivial $L^{\prime}$-automorphism of $L$, which gives $\left[L: \mathbb{F}_{q}(x)\right] \geq 8$ and hence, $P_{0}$ is irreducible over $\mathbb{F}_{q}(x)$, which concludes the proof.

We can again give concrete bounds. With the notations of $\S 3.2$, we have $d=2, m=12, s=8, g_{K}=1$ and there is exactly one ramified point corresponding to $x=0$. The Riemann-Hurwitz formula then gives 
$g_{L} \leq 2$, and thus:

$$
\left|N-\frac{3}{4} q\right| \leq 21 q^{1 / 2}+54 q^{1 / 4}+42
$$

In particular, for $q>2^{16}$ we get

$$
\left|N-\frac{3}{4} q\right| \leq 25 q^{1 / 2}
$$

\section{$5 \quad$ Analogue for the simplified Shallue-Woestijne-Ulas algorithm}

The first deterministic algorithm for hashing to elliptic curves was introduced by Shallue and van de Woestijne in [12]. It was later generalized and simplified by Ulas in [14]. Brier et al. [4] describe a further simplification of the Shallue-Woestijne-Ulas (SWU) algorithm for elliptic curves over fields $\mathbb{F}_{q}$ with $q \equiv 3(\bmod 4)$, based on the following result.

Theorem 2 ([4], Th. 5). Let $\mathbb{F}_{q}$ be a finite field and $g(x):=x^{3}+a x+b$, where $a b \neq 0$. Consider the following rational functions.

$X_{2}(u)=-\frac{b}{a}\left(1+\frac{1}{u^{4}-u^{2}}\right), \quad X_{3}(u)=-u^{2} X_{2}(u), \quad Z(u)=u^{3} g\left(X_{2}(u)\right)$

Then we have $Z(u)^{2}=-g\left(X_{2}(u)\right) \cdot g\left(X_{3}(u)\right)$.

If $q \equiv 3(\bmod 4),-1$ is a quadratic non-residue in $\mathbb{F}_{q}$. Therefore, for each $u$, exactly one of $g\left(X_{2}(u)\right)$ and $g\left(X_{3}(u)\right)$ is a square. This leads to the following deterministic algorithm mapping elements in $\mathbb{F}_{q}$ to points on the curve $E_{a, b}: y^{2}=x^{3}+a x+b$.

\section{Simplified SWU algorithm.}

Input: $\mathbb{F}_{q}$ such that $q>3$ and $q \equiv 3(\bmod 4)$, parameters $a, b$ such that $a b \neq 0$, and input $u \in \mathbb{F}_{q}$.

Output: $(x, y) \in E_{a, b}\left(\mathbb{F}_{q}\right)$.

1. $\alpha \leftarrow-u^{2}$

2. $X_{2} \leftarrow-\frac{b}{a}\left(1+\frac{1}{\alpha^{2}+\alpha}\right)$

3. $X_{3} \leftarrow \alpha \cdot X_{2}$

4. $h_{j} \leftarrow X_{j}^{3}+a X_{j}+b, j=2,3$

5. If $h_{2}$ is a square, return $\left(X_{2}, h_{2}^{(q+1) / 4}\right)$; otherwise, return $\left(X_{3},-h_{3}^{(q+1) / 4}\right)$. 
This algorithm is a slightly modified version of the one described in [4] $\$ 5.5$. The only difference is the minus sign in $\left(X_{3},-h_{3}^{(q+1) / 4}\right)$, which ensures that, up to three possible exceptions (points with a zero $x$-coordinate), the set of points obtained when $g\left(X_{2}(u)\right)$ is a square is disjoint from the set of points obtained when $g\left(X_{3}(u)\right)$ is a square (which improves the size of the image over the original version). Thus, the image of this function $\mathbb{F}_{q} \rightarrow E_{a, b}\left(\mathbb{F}_{q}\right)$ is the (almost disjoint) union of the sets $I_{2}$ and $I_{3}$ defined by

$$
I_{j}=\left\{(x, y) \in E_{a, b}\left(\mathbb{F}_{q}\right) \mid \exists u \in \mathbb{F}_{q}, x=X_{j}(u) \text { and } y=(-1)^{j} \sqrt{g(x)}\right\}
$$

(where $\sqrt{\cdot}$ denotes the standard square root in $\mathbb{F}_{q}$, obtained by exponentiation by $(q+1) / 4)$. Again disregarding at most three points, $I_{j}$ consists of half the points on the curve with an $x$-coordinate of the form $X_{j}(u)$ for some $u$. Therefore, if $N$ is the number of points in the image of the algorithm and $N_{j}$ denotes the number of points with an $x$-coordinate of the form $X_{j}(u)$, we get

$$
N=\frac{N_{2}+N_{3}}{2}+O(1)
$$

and the implied constant is at most 6 . We deduce the following result.

Proposition 4. The number of points $N$ in the image of the simplified $S W U$ algorithm satisfies:

$$
N=\frac{3}{8} q+O\left(q^{1 / 2}\right)
$$

where the implied constant in the big-O is universal.

Proof. The proof is again similar to the previous ones. What we actually show is that $N_{j}=(3 / 8) q+O\left(q^{1 / 2}\right)$ for $j=2,3$, using the Chebotarev density theorem again. Note that for all $u \in \mathbb{F}_{q} \backslash\{-1,0,1\}$, we have

$$
\begin{aligned}
& x=X_{2}(u) \Longleftrightarrow u^{4}-u^{2}+\frac{1}{\omega}=0 \\
& x=X_{3}(u) \Longleftrightarrow u^{4}-\omega u^{2}+\omega=0
\end{aligned}
$$

where $\omega=\frac{a}{b} x+1$. Hence, denoting by $K=\mathbb{F}_{q}(x, y)$ the function field of $E_{a, b}$, it suffices to prove that the polynomials $P_{2}(u)=u^{4}-u^{2}+1 / \omega$ and $P_{3}(u)=u^{4}-\omega u^{2}+\omega$ are irreducible and have Galois group $D_{8}$ (the 8-element dihedral group, viewed as a transitive subgroup of $S_{4}$ ) over 
$K$. Indeed, $D_{8}$ has 8 elements, 3 of which have a fixed point: the same technique as in $\S 3.2$ then gives the desired estimates for $N_{2}$ and $N_{3}$.

In view of [11, Theorems 2 and 3], a polynomial $P(u)=u^{4}-r u^{2}+s \in$ $K[u]$ is irreducible with Galois group $D_{8}$ if and only if none of $s, \delta=r^{2}-4 s$ or $s \delta$ are squares in $K$. For $P_{2}$, we have $(s, \delta, s \delta)=\frac{1}{\omega^{2}}(\omega, \omega(\omega-4), \omega-4)$, and for $P_{3},(s, \delta, s \delta)=\left(\omega, \omega(\omega-4), \omega^{2}(\omega-4)\right)$. Thus, all we have to prove is that $\omega, \omega-4$ and $\omega(\omega-4)$ are not squares in $K$. This is obvious in $\mathbb{F}_{q}(x)$ (since these are polynomials of $\mathbb{F}_{q}[x]$ which are not square), and extends to $K$ by a ramification argument as in the proof of Lemma 2 .

\section{Conclusion}

In this paper, we provide a technique to analyze the image of some hash functions mapping elements of $\mathbb{F}_{q}$ to elliptic curves $E\left(\mathbb{F}_{q}\right)$. It relies on the Chebotarev density theorem in function fields, and in order to apply it, we need to prove the irreducibility of some related polynomial and compute its Galois group.

The same technique should apply similarly to any deterministic, algebraic hash function to curves of any genus. Depending on the particular hash function under consideration, the Galois group varies and the Chebotarev density theorem yields different results accordingly.

\section{Acknowledgements}

We would like to thank Jean-Sébastien Coron and Thomas Icart for suggesting this problem to us, and both of them as well as Éric Brier, Reza Farashahi, Igor Shparlinski, Go Yamamoto and an anonymous referee for helpful comments. The work described in this paper has been supported in part by the European Commission through the ICT program under contract ICT-2007-216676 ECRYPT II.

\section{References}

1. D. Boneh, M.K. Franklin, Identity-based encryption from the Weil pairing, Proceedings of Crypto 2001, LNCS, vol. 2139, Springer-Verlag, 2001, pp. 213-229.

2. C. Boyd, P. Montague, K.Q. Nguyen, Elliptic curve based password-authenticated key exchange protocols, Proceedings of ACISP 2001, LNCS, vol. 2119, SpringerVerlag, 2001, pp. 487-501.

3. V. Boyko, P.D. MacKenzie, S. Patel, Provably secure password-authenticated key exchange using Diffie-Hellman, Proceedings of Eurocrypt 2000, LNCS, vol. 1807, Springer-Verlag, 2000, pp. 156-171. 
4. E. Brier, J.-S. Coron, T. Icart, D. Madore, H. Randriam, M. Tibouchi, Efficient indifferentiable hashing to elliptic curves, Proceedings of CRYPTO 2010, LNCS, Springer-Verlag, 2010, to appear.

5. K. Conrad, Galois groups of cubics and quartics in all characteristics, available at http://www.math.uconn.edu/ kconrad/blurbs/galoistheory/ cubicquarticchar2.pdf

6. D.A. Cox, Galois theory, Series in Pure Mathematics, Wiley, 2004.

7. M.D. Fried, M. Jarden, Field arithmetic, Second edition, Ergebnisse der Mathematik und ihre Grenzgebiete, vol. 11, Springer-Verlag, 2002.

8. C. Gentry, A. Silverberg, Hierarchical ID-based cryptography, Proceedings of Asiacrypt 2002, LNCS, vol. 2501, Springer-Verlag, 2002, pp. 548-566.

9. R.R. Farashahi, I. Shparlinski, J.F. Voloch, On hashing into elliptic curves, J. Math. Crypt. vol. 3(10), 2009, pp. 353-360.

10. T. Icart, How to hash into elliptic curves, Proceedings of Crypto 2009, LNCS, vol. 5677, Springer-Verlag, 2009, pp. 303-316.

11. L.C. Kappe, B. Warren, An elementary test for the Galois group of a quartic polynomial, Amer. Math. Monthly, vol. 96(2), Mathematical Association of America, 1989, pp. 133-137.

12. A. Shallue and C. van de Woestjine, Construction of rational points on elliptic curves over finite fields, Proceedings of ANTS 2006, LNCS, vol. 4076, SpringerVerlag, 2006, pp. 510-524.

13. M. Skałba, Points on elliptic curves over finite fields, Acta Arith., vol. 117, IMPAN, 2005, pp. 293-301.

14. M. Ulas, Rational points on certain hyperelliptic curves over finite fields, Bull. Polish Acad. Sci. Math., vol. 55, IMPAN, 2007, pp. 97-104.

\section{A Galois groups of quartics}

In this appendix, we recall some classical results regarding the computation of Galois groups of quartic polynomials. The reader is refered to texts like [6, Theorem 13.1.1] and [11] for details.

Let $F$ be any field of odd characteristic, and $P(x)=x^{4}+a_{1} x^{3}+$ $a_{2} x^{2}+a_{3} x+a_{4} \in F[x]$ an irreducible polynomial of degree 4 . Let further $\Delta \in F$ be its discriminant, and

$$
C(x)=x^{3}-2 a_{2} x^{2}+\left(a_{2}^{2}+a_{1} a_{3}-4 a_{4}\right) x+\left(a_{3}^{2}+a_{1}^{2} a_{4}-a_{1} a_{2} a_{3}\right)
$$

its resolvent cubic. Then the Galois group $G$ of $P$ is conjugate to:

- $S_{4}$ if $C$ is irreducible and $\Delta$ is not a square in $F$;

- $A_{4}$ if $C$ is irreducible and $\Delta$ is a square in $F$;

- $V_{4}=\mathbb{Z} / 2 \mathbb{Z} \times \mathbb{Z} / 2 \mathbb{Z}$ if $C$ is reducible and $\Delta$ is a square in $F$;

- $D_{8}$ or $\mathbb{Z} / 4 \mathbb{Z}$ otherwise.

If $P$ is a separable polynomial over a field $F$ of characteristic 2, a similar result holds [5, Theorem 3.4], except that the condition that $\Delta$ 
is a square must be replaced by the reducibility over $F$ of the resolvent quadratic $Q$ of $P$, defined by

$Q(x)=x^{2}+\left(a_{1}^{2} a_{4}+a_{1} a_{2} a_{3}+a_{3}^{2}\right) x+\left(a_{1}^{4} a_{4}^{2}+a_{1}^{3} a_{3}^{3}+a_{1}^{2} a_{2}^{3} a_{4}+a_{2}^{3} a_{3}^{2}+a_{3}^{4}\right)$

Finally, when $P$ is an irreducible biquadratic polynomial (i.e. $a_{1}=$ $a_{3}=0$ ) in odd characteristic, its Galois group can be determined by inspection of its coefficients. It is conjugate to:

$-V_{4}$ if $a_{4}$ is a square in $F$;

- $\mathbb{Z} / 4 \mathbb{Z}$ if $a_{4}\left(a_{2}^{2}-4 a_{4}\right)$ is a square in $F$;

- $D_{8}$ if neither $a_{4}$ nor $a_{4}\left(a_{2}^{2}-4 a_{4}\right)$ are squares in $F$. 